\title{
Beyond amyloid: a diverse portfolio of novel drug discovery programs for Alzheimer's disease and related dementias
}

\author{
Rachel F Lane, Diana W Shineman and Howard M Fillit*
}

\begin{abstract}
Although the molecular mechanisms underlying the pathogenesis of Alzheimer's disease and other related neurodegenerative diseases remain unclear, accumulation of misfolded proteins, neuroinflammation, mitochondrial dysfunction and perturbed calcium homeostasis have been identified as key events leading to neuronal loss during neurodegeneration. Evidence for 'druggable' targets for each of these key mechanisms was presented by the Alzheimer's Drug Discovery Foundation-funded investigators at the 12th International Conference on Alzheimer's Drug Discovery, Jersey City, NJ, 26-27 September 2011 (http://www.worldeventsforum.com/ addf/addrugdiscovery).
\end{abstract}

A wide variety of novel therapeutic strategies were presented at the 12th International Conference on Alzheimer's Drug discovery hosted by the Alzheimer's Drug Discovery Foundation (ADDF). This report highlights novel strategies funded by the ADDF that are progressing through the drug development pipeline (Figure 1).

\section{Neuroprotective strategies}

Therapies that are neuroprotective or neurotrophic and can protect against neuronal loss are attractive targets in preventing or slowing the progression of Alzheimer's disease (AD). Carmela Abraham (Boston University School of Medicine, USA) discussed the development of modulators of Klotho expression, a protein that has been shown to decrease with age and regulate maturation of myelinating oligodendrocytes. Three hit compounds were identified from a high-throughput screen (HTS) of 150,000 compounds that inhibit methylation at CpG

*Correspondence: HFillit@alzdiscovery.org

Alzheimer's Drug Discovery Foundation, 57 W57th Street, Suite 904, New York, NY 10014, USA islands and thereby increase Klotho expression. Hits have been validated in several cell lines and are now undergoing a medicinal chemistry strategy to ensure a desirable pharmacological profile. A second neuroprotective strategy was highlighted by Eugenia Trushina (Mayo Clinic College of Medicine, USA). Trushina and team identified and characterized an orally available and blood brain barrier (BBB) permeable tricyclic pyrone, CP-2, which eliminates $80 \%$ of amyloid deposits (fibrillar and oligomers) in the $5 \mathrm{x}$ familial AD (FAD) transgenic model. Probing the mechanism of action revealed metabolomic changes and altered mitochondrial trafficking and function prior to the onset of memory and neurological phenotypes, all of which were reversible with CP-2 treatment.

Other novel neuroprotective strategies targeted neuroinflammation through the orally available tetramethylpyrazine (TMP), derived from a Chinese medical herb (Ziqun Tan, University of California, USA). Martin Watterson (Northwestern University, USA) presented the development program for MW151, a small molecule modulator of $\mathrm{p} 38 \alpha$ mitogen-activated protein kinase (MAPK) activity in neuronal and glial cells with 'druggability' at the forefront of development. Watterson and colleagues undertook a strategic medicinal chemistry program to address the major barriers to $\mathrm{AD}$ therapeutics: central nervous system penetrance and undesired pharmacology. This program resulted in the development of MW151, a highly selective, BBB penetrable compound that effectively attenuated pro-inflammatory cytokine production and reversed behavioral deficits following oral administration in an amyloid- $\beta$-induced brain injury mouse model.

Robert Mahley (University of California, USA) presented an update on PY101, a small molecule that restores apolipoprotein E4 (ApoE4) to an ApoE3-like structure. Ten-day oral administration of PY101 in the neuronspecific enolase ApoE4 (NSE-ApoE4) mouse model resulted in decreased production of toxic ApoE fragments and increased mitochondrial cyclooxygenase-1 levels in the hippocampus. Identification of this toxic gain of function for ApoE4 opens up additional targets in 
this pathway, including protease inhibitors to prevent neuron-specific proteolysis of ApoE4 and mitochondrial protectors to prevent ApoE4 fragment interaction with mitochondria. Finally, the most advanced of these neuroprotective strategies, in phase II/III for the orphan neurodegenerative disease progressive supranuclear palsy (PSP), was presented by Illana Gozes (Allon Therapeutics, USA). Allon Therapeutics' drug (davunetide), a short eight amino acid fragment of activity dependent neurotrophic factor (ADNF), met limited efficacy endpoints in a phase II AD trial, but is now being tested in an improved formulation for PSP.

\section{Calcium homeostasis and plasticity}

Histone deacetylase (HDAC) 2 has been previously implicated in memory formation and synaptic plasticity; however, the development of HDAC2-specific inhibitors has been challenging. To reduce the toxicity associated with this class of compounds, Pavel Petukhov (University of Illinois, USA) screened compounds using Binding Ensemble Profiling with Photoaffinity Labeling to specifically identify compounds that bind in an orientation that prevents chelation of the zinc group. This screen identified a lead compound with an improved pharmacokinetic and toxicity profile.

Altered calcium homeostasis, specifically increased endoplasmic reticulum calcium release, has been linked to a number of sporadic AD risk factors and is thought to underlie altered synaptic transmission and plasticity. Grace Stutzmann (Rosalind Franklin University School of Medicine, USA) and colleagues were the first to observe that the endoplasmic reticulum-resident ryanodine receptor (RyR) differentially regulates synaptic transmission and plasticity in non-transgenic and AD transgenic mice. Chronic treatment of 3x FAD transgenic mice with the RyR antagonist dantrolene (injection) normalized calcium signaling by preventing increased endoplasmic reticulum RyR-mediated calcium release, validating RyR as a potential target for further screening.

Increased intracellular calcium levels affect numerous cellular functional processes via calcium-sensitive proteins. Visinin-like protein 1 (VILIP1), a neuronal calcium sensor protein discovered by Karl-Heinz Braunewell (Southern Research Institute, USA) regulates the cell surface localization of $\alpha 4 \beta 2$ nicotinic acetylcholine receptors. VILIP1 receptors are decreased in the hippocampus, amygdala and cortical areas of AD patients. A phenotypic and biochemical HTS of 2,000 compounds identified 2 lead compounds that will now be optimized for the desired pharmacological properties.

\section{Protein folding and degradation}

Heat shock proteins (HSPs) promote the correct folding and refolding of misfolded proteins, including tau, amyloid- $\beta$, and $\alpha$-synuclein, and are therefore attractive targets for a number of neurodegenerative diseases. Several approaches to target HSP70 and HSP90 were presented. Allen Reitz (ALS Biopharma, USA) identified hit compounds that lowered tau levels by inducing HSP70 expression in human glioblastoma cell lines. Understanding the mechanism of action of a target is vitally important during drug design. This point was highlighted by Chad Dickey (University of South Florida, USA), who discovered that inhibition of HSP70 ATPase activity could also be effective at stabilizing disorganized tau. Inhibitors from a HTS based on rhodacyanine dyes have now been validated for their ability to decrease tau accumulation and rescue long-term potentiation in hippocampal slice cultures from Tg4510 tau mice. A second member of the heat shock family, HSP90, was the focus of Gabriela Chiosis's (Memorial Sloan Kettering Cancer Center, USA) drug discovery program. Chiosis identified two populations of HSP90 in pathogenic or stressed tissue (tumors and dystrophic neurons); a good housekeeping HSP90 complex and an HSP90 complex that selectively binds to pathogenic protein. Hsp90 inhibitors developed by this team specifically bind the pathogenic HSP90 complex and reduce total tau and phospho-tau following acute treatment in the 3x FAD transgenic mouse model. Chronic studies will be required to assess behavioral outcomes.

Dysfunctional protein degradation pathways contribute to neurodegeneration. Karen Duff (Columbia University, USA) provided target validation for autophagy and proteasome targets demonstrating that mice with progressive tauopathy show a decline in proteasome function concomitant with an induction of autophagy and accumulation of fibrillar tau aggregates. In vivo induction of autophagy following acute oral administration of trehalose, a non-reducing dissacharide, effectively decreased tau pathology in the P301L tau mouse model. Duff noted that the decreased proteosomal function seen during the onset of pathology could be reversed pharmacologically, highlighting both autophagy and proteasome activation as two potential drug targets in stimulating clearance of protein aggregates that present in numerous neurodegenerative diseases.

Lysosomal function plays a critical role in protein clearance. Lysosomal storage diseases, such as Gaucher disease, Fabry disease and Sandhoff disease, show evidence of accumulated intraneuronal amyloid- $\beta$ in addition to decreased lysosome enzyme function. Brandon Wustman (Amicus Therapeutics, USA) and team have developed a set of novel pharmacological chaperones that enhance the activity of critical lysosomal enzymes, are orally available, cross the BBB, and are currently in phase III for Fabry disease. Now, Wustman and team are testing a novel, potent pharmacological chaperone for its effects in 


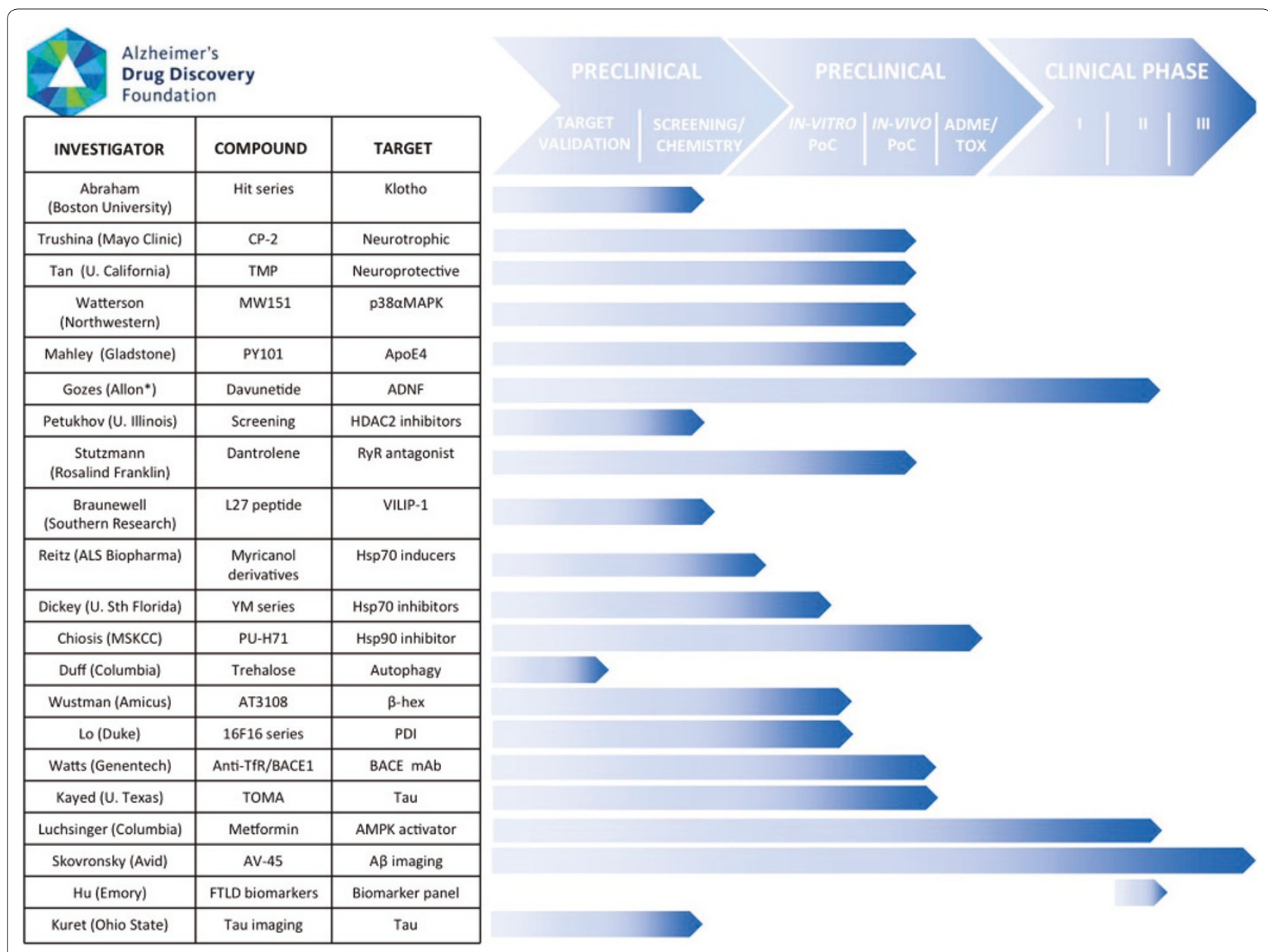

Figure 1. Development timeline of Alzheimer's Drug Discovery Foundation-funded therapeutic strategies for Alzheimer's disease and related dementias. Each program presented at the 12th International conference on Alzheimer's Drug Discovery is highlighted in terms of position in the drug development lifecycle at the time of the meeting. *Allon Theraputics is currently in phase II/III for progressive supranuclear palsy, following limited efficacy in a phase II trial of davunetide for Alzheimer's disease. NB: the purpose of this figure is to highlight the stage of development for each program that has benefited from Alzheimer's Drug Discovery Foundation funding, and is not indicative of Alzheimer's Drug Discovery Foundation funding throughout the entire program lifecycle. Multiple funders have contributed to many of these programs. $A \beta$, amyloid- $\beta$; ADME, absorption, distribution, metabolism and excretion; ADNF, activity dependent neurotrophic factor; AMPK, AMP activated protein kinase; APOE, apolipoprotein E; BACE, $\beta$-site APP cleaving enzyme; FTLD, frontotemporal lobar degeneration; HDAC, histone deacetylase; HSP, heat shock protein; mAb, monoclonal antibody; MAPK, mitogen-activated protein kinase; MSKCC, Memorial Sloan Kettering Cancer Center; PDI, protein disulphide isomerase; PoC, proof of concept; RyR, ryanodine receptor; TfR, transferrin receptor; TMP, tetramethylpyrazine; TOMA, tau oligomer monoclonal antibody; TOX, toxicity; VILIP, Visinin-like protein.

clearing intraneuronal amyloid- $\beta$ in an animal model of cerebral amyloid angiopathy, an orphan indication with relevance to $\mathrm{AD}$.

Additional strategies at the proof-of-concept stage targeted protein aggregates through immunotherapy. Rakez Kayed (University of Texas, USA) has developed an oligomer immunotherapy agent, TOMA (tau oligomer monoclonal antibody), that effectively reduced tau pathology following intracerebroventricular injection in the P301L tau mouse model. In the plenary session, Ryan Watts (Genentech, USA) demonstrated that $\beta$-site APP cleaving enzyme (BACE) monoclonal antibodies engineered to cross the $\mathrm{BBB}$ via the transferrin receptor effectively reduced BACE activity and amyloid- $\beta$ levels without affecting total BACE expression in an AD mouse model.

\section{Novel biomarkers}

The development of novel biomarkers to accurately diagnose $\mathrm{AD}$ prior to clinical manifestation and to predict conversion from mild cognitive impairment to $A D$ is essential for the development of new therapeutics and understanding mechanisms of disease progression. Currently, the only definitive diagnostic tool for AD is at autopsy. The most developmentally advanced imaging 
biomarker, AV-45 (florbetapir), is currently up for approval by the US Food and Drug Administration (FDA). Daniel Skovronsky (Avid Radiopharmaceuticals, Inc., USA), funded by the Alzheimer's Drug Discovery Foundation (ADDF) for the initial stages of development of florbetapir-positron emission tomography (PET), provided the opening plenary address. Florbetapir ${ }^{18} \mathrm{~F}$ is a PET imaging agent with high specificity for amyoid- $\beta$ binding. Skovronksy presented data from longitudinal analysis of normal older people with positive florbetapirPET scans and identified a correlation between scan positivity and cognitive decline in normal older people, providing further evidence that amyloid- $\beta$ load correlates with cognitive function.

Diagnostic tests that correctly diagnose different causes of dementia are clinically challenging and remain a critical unmet need. Biomarkers that efficiently distinguish between frontotemporal lobar degeneration (FTLD) with TDP43 (TAR DNA-binding protein 43) or tau pathology are necessary for the development of disease-modifying treatments and to identify patients for inclusion/ exclusion from clinical trials. William $\mathrm{Hu}$ (Emory University School of Medicine, USA) provided an update on the program to identify relevant biomarker(s) using a targeted proteomics approach. $\mathrm{Hu}$ and colleagues identified a panel of analytes from cerebrospinal fluid samples that potentially distinguish between FTLD with TDP43 pathology and FTLD with tau pathology. The team is currently recruiting patients for a multi-center study to validate this panel. Jeff Kuret (Ohio State University, USA) presented data on a HTS to identify selective radiolabeled compounds with high selectivity for tau by utilizing amino acid side chains of cross- $\beta$ sheet aggregates to increase binding selectivity. The team has identified potent lead candidates currently under optimization.

Allen Roses (Duke University, USA) highlighted the utility of biomarkers to enrich patient populations in clinical trials. Roses and team have identified a region on chromosome 19 next to $A P O E$ that encodes the mitochondrial protein TOMM40 (translocase of outer mitochondrial membrane 40 homolog) and can help explain the age-of-onset variability within the $A P O E$ genotype. Through an algorithm-based approach, Roses and team are testing the prognostic value of the TOMM40 genotype, $A P O E$ variants and age for patient inclusion in a trial of pioglitazone in normal subjects. To close the program, José Luchsinger (Columbia University, USA) presented the use of $\left.{ }^{18}\right]$ F-labeled 2-deoxy-2-fluoro-Dglucose (FDG) PET with magnetic resonance imaging as a secondary endpoint for an ongoing phase II clinical trial for the anti-diabetic metformin in patients with mild cognitive impairment.

The ADDF drug discovery portfolio encompasses a wide spectrum of novel targets in the generation of therapeutics for the treatment of $\mathrm{AD}$ and related dementias. The work presented highlights the innovative approaches required during preclinical and clinical development. The ADDF will continue to support novel targets in order to achieve our mission to conquer $\mathrm{AD}$ through drug discovery. Please join us for our 13th annual International Conference on Alzheimer's Drug Discovery in the Fall of 2012 in Jersey City, NJ, USA.

\section{Abbreviations}

AD, Alzheimer's disease; ADDF, Alzheimer's Drug Discovery Foundation; APOE, apolipoprotein E; BACE, $\beta$-site APP cleaving enzyme; BBB, blood brain barrier; FAD, familial Alzheimer's disease; FTLD, frontotemporal lobar degeneration; HDAC, histone deacetylase; HSP, heat shock protein; HTS, high-throughput screen; PET, positron emission tomography; PSP, progressive supranuclear palsy; RyR, ryanodine receptor; TDP43, TAR DNA-binding protein 43; TOMM40, translocase of outer mitochondrial membrane 40 homolog.

\section{Competing interests}

The authors declare that they have no competing interests.

\section{Acknowledgements}

This conference would not have been possible without the generous support of our sponsors: Pfizer Inc., Eli Lilly and Company, Elan Pharmaceuticals, Merck Research Laboratories, Allon Therapeutics, NeuroPhage Pharmaceuticals, JSW Life Sciences, Abbott Laboratories and Genentech.

Published: 20 December 2011

\section{doi:10.1186/alzrt99}

Cite this article as: Lane RF, et al:: Beyond amyloid: a diverse portfolio of novel drug discovery programs for Alzheimer's disease and related dementias. Alzheimer's Research \& Therapy 2011, 3:36. 